\title{
Interactive comment on "Observation of an optical anisotropy in the deep glacial ice at the geographic South Pole using a laser dust logger" by Martin Rongen et al.
}

Martin Rongen et al.

rongen@physik.rwth-aachen.de

Received and published: 15 May 2020

Dear Jan Eichler,

Thank you for your timely review. Please find the responses to the issues raised in-line with your review comments below: 
tered light in ice. The scattering anisotropy was measured using a laser dust logger deployed in the SPICEcore borehole. The study follows previous observations of anisotropic light propagation in ice at the IceCube observatory. The measurements support results obtained from simulations of light diffusion by Chirkin and Rongen (2019). The authors conclude that scattering due to reflection and refraction on grain boundaries in a birefringent polycrystal play a major role for the anistropic modulation of light in ice.

The study is carefully carried out and well presented. It shows novel in-situ measurements of optical properties of ice. I believe it is a relevant contribution to our understanding of light propagation and scattering in ice. It may find application in current ice-core-analytical methods as well as in future developments.

I have some concerns about the general suitability of the method for obtaining a continuous fabric record. The intensity ratios can serve to determine the strength of anisotropy within a horizontal plane, so it works well for a girdle-type fabric. However, other types of arrangements of the c-axes can hardly be distinguished or even detected using the current design of the dust logger (e. g. a vertical single maximum, or other more complex distributions).

\section{Specific comments:}

p.1 1.7-8: See the last paragraph in general comments. I think the statement is too brave. Maybe it is just the term "fabric" which, in my understanding, includes a number of characteristics, which I am not convinced they are accessible for the dust logger.

We agree with the assessment, that the current data will most likely 
not be able to unambiguously constrain the many fabric properties. The requirements to study this possibility (photon propagation simulation of the experimental setup), as well as potential improvements to the experimental data (to include the measurement of propagation delays), are detailed in the last paragraph of section 6 .

The sentence in the abstract has been changed to:

In the future, the measurement principle, when combined with a full-chain simulation, may have the potential to provide a continuous record of fabric properties along the entire depth of a drill hole.

p.2 I.16-17: What crystal realization would strengthen the deflection effect?

A stronger deflection effect would result from a stronger girdle, a larger mean crystal elongation as well as a smaller overall crystal size.

The paragraph has been expanded to read as follows (also taking into account comments from the other reviewer):

The deflection per distance increases for stronger girdle fabrics, a larger average crystal elongation or a smaller average crystal size. For crystal realizations where the deflection outweighs the additional diffusion along the flow axis compared to the diffusion along the orthogonal direction, the photon flux along the flow axis will increase with distance compared to the photon flux along the orthogonal axis. 
p.4 I.11-12: The corresponding accumulation site varies with depth, so the Titan Dome must refer to a particular depth in the ice core.

That's a good point (as also raised by the other reviewer). The sentence has been changed to:

The associated accumulation site for the deepest ice is believed by

? to be Titan Dome, meaning that the ice has been transported as far as $200 \mathrm{~km}$.

p.6 I.10 and p.10 I.8: "craigite": The common terms are clathrate hydrates or air hydrates.

The term has been changed to "clathrate hydrate".

p.9 Fig.7: If there is no anisotropy signature above $1100 \mathrm{~m}$, how can you determine the anisotropy axis being 120 degrees above this depth?

Thank you very much for raising this issue! We had originally assumed that the correctly fitted axis might be an indication for a small residual anisotropy signature. This is not the case. We have instead traced the problem to a minimizer artifact. The seed value for the angle was originally chosen to be $120^{\circ}$. In the absence of a significant signal, the fit values seem to be pulled towards the seed value instead of scattering randomly. Re-seeding the minimizer with different anisotropy axis directions did not significantly change the measured anisotropy strength or axis below $1100 \mathrm{~m}$ (within the uncertainty). 
The original paragraph read as:

Individual ratios are seen to yield consistent anisotropy axes in the deep ice. In the shallow ice above $\sim 1100 \mathrm{~m}$, where the mean strength of the observed anisotropy signal vanishes, the phase angle is badly constrained and varies widely between individual ratios. The per-depth average axes are compatible with the global average of $126 \pm 1$ (stat) $^{\circ}$.

This will be changed to:

Individual ratios are seen to yield consistent anisotropy axes in the deep ice. In the shallow ice above $\sim 1100 \mathrm{~m}$, where the mean strength of the observed anisotropy signal vanishes, the phase angle is unconstrained. The average axis in the deep ice is $126 \pm 1$ (stat) ${ }^{\circ}$.

Attached please find a figure showing the impact of the seed value as well as the plot to be used in the updated version of the manuscript.

p.10 I.10-16: I also wonder what would be the effect of the grain shape and its preferred orientation. Also the scattering on grain boundary intersections (triple junctions) may add some contribution - possibly even an anisotropic one.

Grain shape after averaging over many thousand encountered crystals is by us assumed to be an ellipsoid with the mayor axis being closely aligned with the flow direction. It is the second major contribution (besides the c-axis distribution) to the effect, as it 
changes the grain boundary orientation distribution. As shape and fabric are strongly linked (in a girdle fabric the average crystal shape has to be elongated) we did not explicitly elaborate on this. We hope this is now covered, by the extended description of the general birefringence effect on page 2 .

Interactive

comment

Triple junctions have so far not been considered. But as their fractional contribution to the surface area of a grain is rather small, their effect should be negligible.

Interactive comment on The Cryosphere Discuss., https://doi.org/10.5194/tc-2020-34, 2020. 


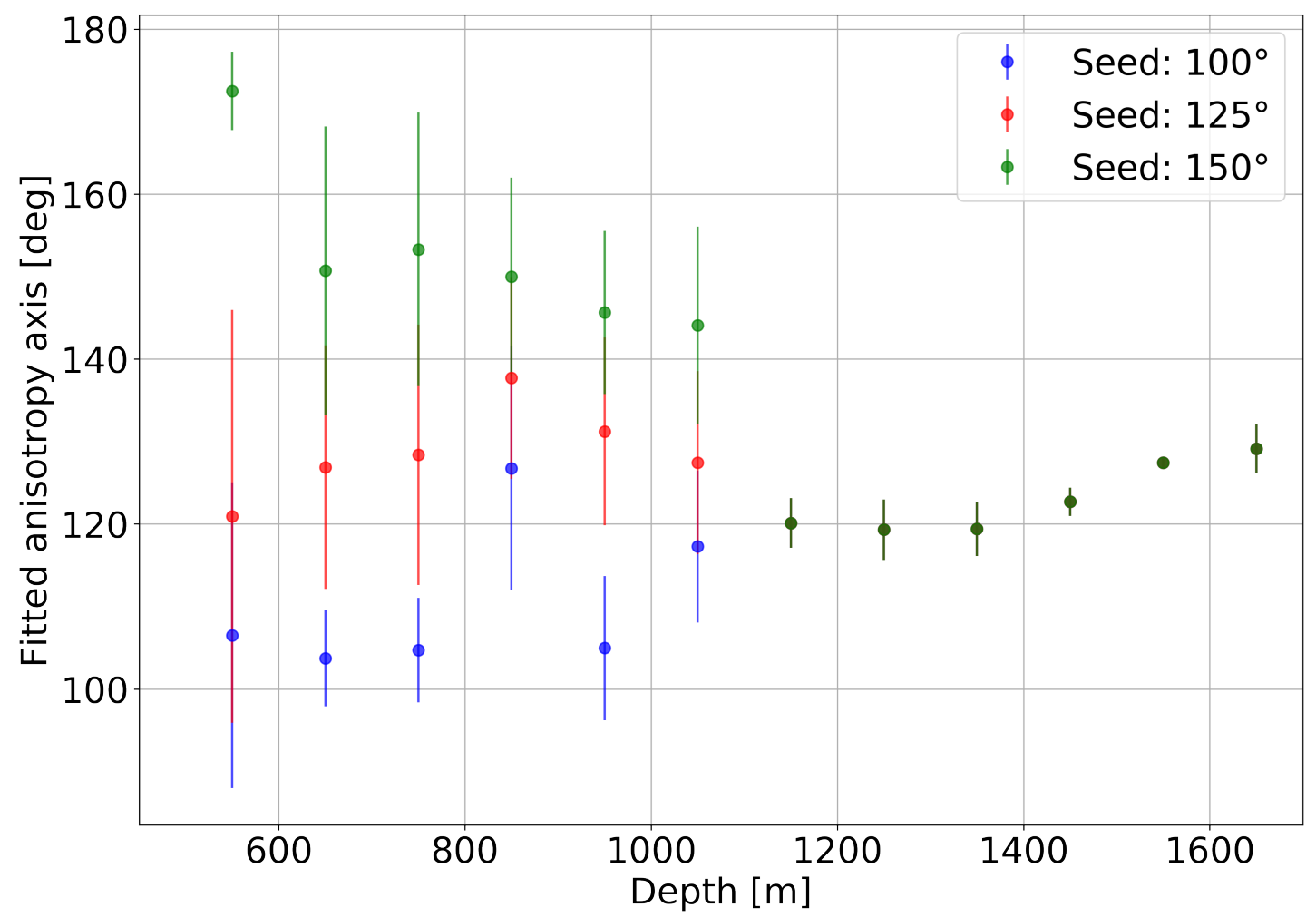

Interactive

comment

Fig. 1. Impact of the minimizer seed on the axis fit. 


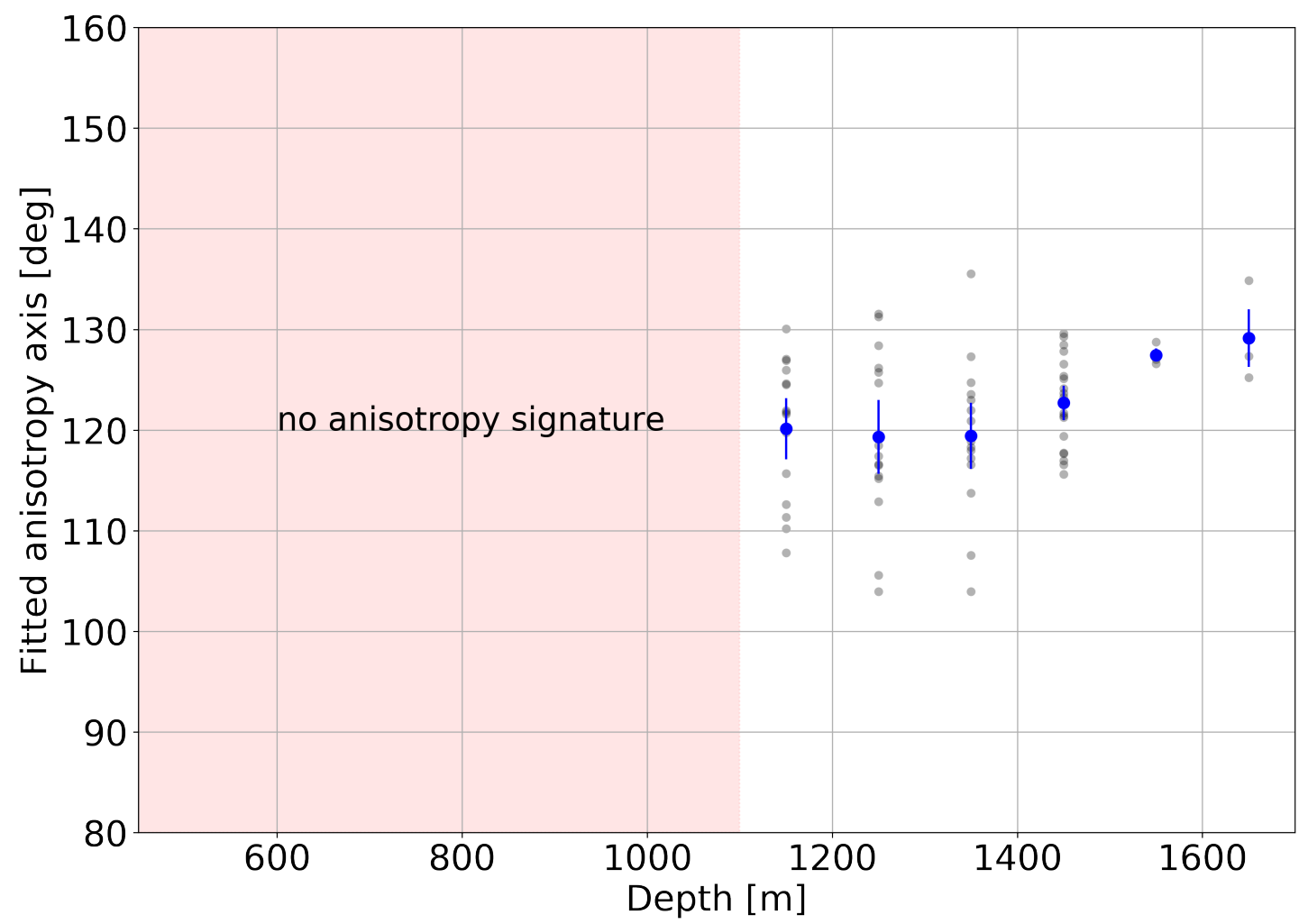

Interactive

comment

Fig. 2. New figure for the depth dependent anisotropy axis. 